\title{
Projection Pursuit Multi-Index (PPMI) Models
}

\author{
Michael G. Akritas \\ Department of Statistics, Penn State University, University Park, PA 16802, USA
}

\begin{abstract}
The concept of joint projective directions and the class of projection pursuit multi-index (PPMI) models are introduced. PPMI models are MI models with hierarchically defined directions spanning the central mean subspace, and bridge the gap between PP and MI models.
\end{abstract}

Keywords:

dimension reduction, first projective direction, joint projective directions, multi-index model, projection pursuit regression

\section{Introduction}

For a univariate response $Y$, and covariate vector $\mathbf{X} \in \mathbb{R}^{d}$, set $\mu(\mathbf{X})=$ $E(Y \mid \mathbf{X})$. Attempts to overcome the "curse of dimentionality" in the nonparametric estimation of $\mu(\mathbf{X})$, led to intensive work on models for highdimensional data which culminated with the works of Huber (1985) and Stone (1985). Thirty years later, it can be said that among the various dimension reduction models proposed, the multi-index (MI) model is probably the most studied. Special cases of the MI model are the projection pursuit regression (PPR) model and the single index (SI) model, which has received particular attention.

The SI model specifies

$$
\mu(\mathbf{X})=g\left(\boldsymbol{\beta}^{T} \mathbf{X}\right),
$$

where the $d \times 1$ vector $\boldsymbol{\beta}$ and function $g$ are unknown. For identifiability, it is assumed either that $\|\boldsymbol{\beta}\|=1$, with $\beta_{1}>0$, where $\beta_{1}$ denotes the fist coordinate of $\boldsymbol{\beta}$, or $\boldsymbol{\beta}=\left(\beta_{1}, \boldsymbol{\beta}_{-1}^{T}\right)^{T}$, with $\left\|\boldsymbol{\beta}_{-1}\right\|<1$ and $\beta_{1}=(1-$ $\left.\left\|\boldsymbol{\beta}_{-1}\right\|^{2}\right)^{1 / 2}$, or $\boldsymbol{\beta}=\left(1, \boldsymbol{\beta}_{-1}^{T}\right)^{T}$; see (7) for the parametrization adopted here. The term single index model was coined by Stoker (1986), though the model 
was first introduced by Brillinger (1983) who also introduced the least squares estimator as first estimator for $\boldsymbol{\beta}$, under a "linearity" condition.

The PPR model, proposed by Friedman and Stuetzle (1981), specifies

$$
\mu(\mathbf{X})=\sum_{k=1}^{K} g_{k}\left(\boldsymbol{\beta}_{k}^{T} \mathbf{X}\right), \text { with } g_{1}, \ldots, g_{k} \text { unknown. }
$$

See Huber (1985) for a comprehensive discussion. The parameters $\boldsymbol{\beta}_{k}$ are called projective directions, and are defined recursively as follows. Assuming the first $k-1$ projective directions, $\boldsymbol{\beta}_{1}, \ldots, \boldsymbol{\beta}_{k-1}$, and corresponding functions $g_{1}, \ldots, g_{k-1}$, have been determined, $\boldsymbol{\beta}_{k}$ and $g_{k}$ are determined by

$$
\boldsymbol{\beta}_{k}=\arg \inf _{\mathbf{b}} E\left[\left(R-E\left(R \mid \mathbf{b}^{T} \mathbf{X}\right)\right)^{2}\right]
$$

where $R=Y-\sum_{i=1}^{k-1} g_{i}\left(\boldsymbol{\beta}_{i}^{T} \mathbf{X}\right)$, and $g_{k}(t)=E\left(R \mid \boldsymbol{\beta}_{k}^{T} \mathbf{X}=t\right)$. Identifiability of the projective directions is similar to that of the SIM. The hierarchy among the projective directions is conceptually appealing, but the forced additivity restricts generality. When $K=1$, the PPR model reduces to the SIM, so that the parameter $\boldsymbol{\beta}$ in (1) equals the first projective direction.

The multi-index model specifies

$$
\mu(\mathbf{X})=g\left(\mathbf{B}^{T} \mathbf{X}\right),
$$

for some unknown function $g$, and unknown full rank matrix $\mathbf{B}$. The MI model (4) is of order $k$ if $\mathbf{B}=\left(\boldsymbol{\beta}_{1}, \ldots, \boldsymbol{\beta}_{k}\right)$, with $\boldsymbol{\beta}_{i} \in \mathbb{R}^{d}$. Identifiability of $\boldsymbol{\beta}_{1}, \ldots, \boldsymbol{\beta}_{k}$ is discussed by several authors; cf. Xia (2008).

A conceptual disconnect between the single and multi-index models has to do with the fact that none of the parametrizations involves the first projective direction. In fact, it is not clear from the literature whether or not the first projective direction lies in the central mean subspace, which is defined as the subspace spanned by the columns of $\mathbf{B}$.

In this paper we propose a new class of multi-index models, called PPMI because of the use of projection pursuit ideas in their definition and parameter identification. The most general member of this class is (under conditions) a multi-index model with a different type of parametrization. This new parametrization starts with the first projective direction and the additional directions are chosen hierarchically according to their relative usefulness. From the modeling standpoint, the desirable feature of the new 
parametrization is that a PPMI model of order $k$ will account for at least as much variability as any MI model of order $k$. Consequently, it is likely that in a model building context, PPMI modeling will result in a more parsimonious model.

\section{The PPMI Model}

For any given $d$-dimensional vectors $\mathbf{b}_{1}, \ldots, \mathbf{b}_{r}, r \leq d$, we define the corresponding $r$-dimensional projective approximation of $\mu(\mathbf{X})$ by

$$
g_{r}\left(u_{1}, \ldots, u_{r} \mid \mathbf{b}_{1}, \ldots, \mathbf{b}_{r}\right)=E\left(Y \mid \mathbf{b}_{1}^{T} \mathbf{X}=u_{1}, \ldots, \mathbf{b}_{r}^{T} \mathbf{X}=u_{r}\right) .
$$

Short hand notations for $g_{r}\left(u_{1}, \ldots, u_{r} \mid \mathbf{b}_{1}, \ldots, \mathbf{b}_{r}\right)$, such as $g_{r}(\mathbf{u} \mid \mathbf{B})$, where $\mathbf{u}=\left(u_{1}, \ldots, u_{r}\right)^{T}$ and $\mathbf{B}$ is the $d \times r$ matrix $\left(\mathbf{b}_{1}, \ldots, \mathbf{b}_{r}\right)$, will also be used.

Define the first projective direction, $\boldsymbol{\vartheta}_{1}$, by

$$
\boldsymbol{\vartheta}_{1}=\arg \inf _{\mathbf{b}} E\left[\left(Y-g_{1}\left(\mathbf{b}^{T} \mathbf{X} \mid \mathbf{b}\right)\right)^{2}\right],
$$

where, for identifiability pursposes, $\boldsymbol{\vartheta}_{1}$ is taken to be of the form

$$
\boldsymbol{\vartheta}_{1}=\left(1, \boldsymbol{\theta}_{1}^{T}\right)^{T}, \boldsymbol{\theta}_{1} \in \mathbb{R}^{d-1} .
$$

Thus, the minimization in (6) is specialized to $\mathbf{b}$ of the form $\mathbf{b}_{\mathbf{t}}=\left(1, \mathbf{t}^{T}\right)^{T}$, $\mathbf{t} \in \mathbb{R}^{d-1}$.

For $r=2, \ldots, d$, define recursively the $r$ th joint projective direction, $\boldsymbol{\vartheta}_{r}$, by

$$
\boldsymbol{\vartheta}_{r}=\arg \inf _{\mathbf{b}} E\left[\left(Y-g_{r}\left(\boldsymbol{\Theta}_{r-1}^{T} \mathbf{X}, \mathbf{b}^{T} \mathbf{X} \mid \boldsymbol{\Theta}_{r-1}, \mathbf{b}\right)\right)^{2}\right]
$$

subject to the parametrization specified below, where $\boldsymbol{\Theta}_{r-1}=\left(\boldsymbol{\vartheta}_{1}, \ldots, \boldsymbol{\vartheta}_{r-1}\right)$. For example, the 2nd joint projective direction is defined as

$$
\boldsymbol{\vartheta}_{2}=\arg \inf _{\mathbf{b}} E\left[\left(Y-g_{2}\left(\boldsymbol{\vartheta}_{1}^{T} \mathbf{X}, \mathbf{b}^{T} \mathbf{X} \mid \boldsymbol{\vartheta}_{1}, \mathbf{b}\right)\right)^{2}\right],
$$

i.e., as the direction which, when taken jointly with $\boldsymbol{\vartheta}_{1}$, accounts for the most variability in $Y$. If

$$
E\left[\left(Y-g_{r}\left(\boldsymbol{\Theta}_{r-1}^{T} \mathbf{X}, \mathbf{b}^{T} \mathbf{X} \mid \boldsymbol{\Theta}_{r-1}, \mathbf{b}\right)\right)^{2}\right]=E\left[\left(Y-g_{r-1}\left(\boldsymbol{\Theta}_{r-1}^{T} \mathbf{X} \mid \boldsymbol{\Theta}_{r-1}\right)\right)^{2}\right]
$$

holds for all $\mathbf{b}$ then the $r$ th joint projective direction is undefined and we can arbitrarily set $\boldsymbol{\vartheta}_{r}=\mathbf{0}$. 
Note that, when non-zero, $\boldsymbol{\vartheta}_{r}$ is well defined up to the space generated by it and the columns of $\boldsymbol{\Theta}_{r-1}$. That is, if $\mathcal{M}(\mathbf{B})$ denotes the subspace spanned by the columns of $\mathbf{B}$, any $\boldsymbol{\vartheta}_{r}^{*}$ such that

$$
\mathcal{M}\left(\boldsymbol{\Theta}_{r-1}, \boldsymbol{\vartheta}_{r}\right)=\mathcal{M}\left(\boldsymbol{\Theta}_{r-1}, \boldsymbol{\vartheta}_{r}^{*}\right)
$$

also minimizes $E\left[\left(Y-g_{r}\left(\boldsymbol{\Theta}_{r-1}^{T} \mathbf{X}, \mathbf{b}^{T} \mathbf{X} \mid \boldsymbol{\Theta}_{r-1}, \mathbf{b}\right)\right)^{2}\right]$. Thus, the search for the minimum can be confined on $\mathcal{M}\left(\boldsymbol{\Theta}_{r-1}\right)^{c}$, the complement of $\mathcal{M}\left(\boldsymbol{\Theta}_{r-1}\right)$. We adopt a parametrization which facilitates the search while also ensuring identifiability. Consider first $\boldsymbol{\vartheta}_{2}$. Given the parametrization (7) for $\boldsymbol{\vartheta}_{1}$, using $\boldsymbol{\vartheta}_{2}$ of the form $\boldsymbol{\vartheta}_{2}=\left(0, \overline{\boldsymbol{\theta}}_{2}^{T}\right)^{T}$, with $\overline{\boldsymbol{\theta}}_{2} \in \mathbb{R}^{d-1}$, ensures that $\boldsymbol{\vartheta}_{2} \in \mathcal{M}\left(\boldsymbol{\vartheta}_{1}\right)^{c}$ and any other vector in $\mathcal{M}\left(\boldsymbol{\vartheta}_{1}\right)^{c}$ can be expressed as a linear combination of $\boldsymbol{\vartheta}_{1}$ and some $\boldsymbol{\vartheta}_{2}$. Finally, to ensure identifiability, one of the coordinates of $\overline{\boldsymbol{\theta}}_{2}$ can be set to 1 . Without loss of generality, we can set $\overline{\boldsymbol{\theta}}_{2}=\left(1, \boldsymbol{\theta}_{2}^{T}\right)^{T}$. In summary, $\boldsymbol{\vartheta}_{2}$ is parametrized as $\boldsymbol{\vartheta}_{2}=\left(0,1, \boldsymbol{\theta}_{2}^{T}\right)^{T}$ and the search for a minimum in (6) to $\mathbf{b}$ of the form $\mathbf{b}_{2, \mathbf{t}}=\left(0,1, \mathbf{t}_{2}^{T}\right)^{T}, \mathbf{t}_{2} \in \mathbb{R}^{d-2}$. In general, $\boldsymbol{\vartheta}_{r}$ will be parametrized as

$$
\boldsymbol{\vartheta}_{r}=\left(\mathbf{0}_{r-1}^{T}, 1, \boldsymbol{\theta}_{r}^{T}\right)^{T}, \boldsymbol{\theta}_{r} \in \mathbb{R}^{d-r},
$$

where $\mathbf{0}_{m}$ denotes an $m$-dimensional column vector of $0 \mathrm{~s}$. Thus, the minimization in (8) can be confined to $\mathbf{b}$ of the form $\mathbf{b}_{r, \mathbf{t}}=\left(\mathbf{0}_{r-1}^{T}, 1, \mathbf{t}_{r}^{T}\right)^{T}$, $\mathbf{t}_{r} \in \mathbb{R}^{d-r}$.

Remark 2.1. The parametrization (7), (10) does not restrict generality, but may require some reordering/renumbering of the covariates. For example, the parametrization for $\boldsymbol{\vartheta}_{1}$ requires that the first covariate enters the first projective direction with a non-zero coefficient, which is a standard requirement. Let now $\boldsymbol{\theta}_{2}^{*}$ be a solution to the minimization problem in (9). The parametrization (10) for $r=2$ is possible provided the second covariate enters $\boldsymbol{\theta}_{2}^{*}$ with a nonzero coefficient, and the coefficients of the fist two covariates in $\boldsymbol{\theta}_{2}^{*}$ are not proportional to their coefficients in $\boldsymbol{\theta}_{1}$. Indeed, if these conditions are satisfied, the parametrization (10) for $r=2$ can be achieved by a linear combination of $\boldsymbol{\theta}_{1}$ and $\boldsymbol{\theta}_{2}^{*}$, and by dividing the coordinates of the linear combination by the second coordinate. For PPMI models of order $\geq 2$ a permutation of the components of the covariate vector that satisfies these conditions always exists. Similarly, the parametrization (10) for $r=3$ is possible provided the third covariate enters a solution $\boldsymbol{\theta}_{3}^{*}$ with a nonzero coefficient, and the coefficients of the fist three covariates in $\boldsymbol{\theta}_{3}^{*}$ are not a 
linear combination of their coefficients in $\boldsymbol{\theta}_{1}$ and $\boldsymbol{\theta}_{2}$. If these conditions are satisfied, the parametrization (10) for $r=3$ can be achieved by a linear combination of $\boldsymbol{\theta}_{1}, \boldsymbol{\theta}_{2}$ and $\boldsymbol{\theta}_{3}^{*}$, and by dividing the coordinates of the linear combination by the third coordinate. And so on.

Definition 1. 1. We say that $(Y, \mathbf{X})$ follows the PPMI $(k)$ (PPMI of order k) model, if

$$
\mu(\mathbf{X})=g_{k}\left(\boldsymbol{\Theta}_{k}^{T} \mathbf{X} \mid \boldsymbol{\Theta}_{k}\right),
$$

where $\boldsymbol{\Theta}_{k}=\left(\boldsymbol{\vartheta}_{1}, \ldots, \boldsymbol{\vartheta}_{k}\right)$, and $k$ is the smallest integer for which $\boldsymbol{\vartheta}_{r} \neq$ $\mathbf{0}, r=1, \ldots, k$.

2. We say that $(Y, \mathbf{X})$ follows the $\operatorname{PPMI}\left(k_{1}, \ldots, k_{s}\right)$ model if it follows the PPMI $(k)$ model with $k=k_{1}+\cdots+k_{s}$, and there exist functions $G_{1}: \mathbb{R}^{k_{1}} \rightarrow \mathbb{R}, \ldots, G_{s}: \mathbb{R}^{k_{s}} \rightarrow \mathbb{R}$ such that $g_{K_{\ell}}\left(u_{1}, \ldots, u_{K_{\ell}} \mid \Theta_{K_{\ell}}\right)=G_{1}\left(u_{1}, \ldots, u_{k_{1}}\right)+\cdots+G_{\ell}\left(u_{K_{\ell-1}}, \ldots, u_{K_{\ell}}\right)$, holds for $\ell=1, \ldots, s$, where $K_{\ell}=k_{1}+\cdots+k_{\ell}$. In particular,

$$
\mu(\mathbf{X})=G_{1}\left(\boldsymbol{\Theta}_{k_{1}}^{T} \mathbf{X}\right)+\cdots+G_{s}\left(\mathbf{X}^{T} \boldsymbol{\Theta}_{K_{s-1}}^{K_{s}}\right),
$$

where $\boldsymbol{\Theta}_{K_{\ell-1}}^{K_{\ell}}=\left(\boldsymbol{\vartheta}_{K_{\ell-1}+1}, \ldots, \boldsymbol{\vartheta}_{K_{\ell}}\right)$.

From Definition 1 it follows that PPR models are $\operatorname{PPMI}(1, \ldots, 1)$ models. Thus, PPMI $\left(k_{1}, \ldots, k_{s}\right)$ models bridge the gap between PPR and PPMI models. It is also clear that PPMI models are MI models with a parametrization that prioritizes the relative importance of the different linear combinations. The following result gives sufficient conditions for a MI model to be parametrized as a PPMI model.

Theorem 1. Assume that $(Y, \mathbf{X})$ follows the MI model (4), and that $\mathbf{X}$ has an elliptically symmetric distribution with positive definite covariance matrix. Then, if all projective directions $\boldsymbol{\vartheta}_{\ell}$ are uniquely defined, we have

$$
\boldsymbol{\vartheta}_{\ell} \in \mathcal{M}(\mathbf{B}) \text {. }
$$

The concept of central mean subspace, introduced in Cook and Li (2002), is particularly relevant for dimension reduction problems when prediction is the primary focus. Theorem 1 yields the following.

Corollary 2.1. Let the assumptions of Theorem 1 hold, let $\boldsymbol{\vartheta}_{1}, \ldots, \boldsymbol{\vartheta}_{k}$ be the non-zero projective directions, and set $\boldsymbol{\Theta}_{k}=\left(\boldsymbol{\vartheta}_{1}, \ldots, \boldsymbol{\vartheta}_{k}\right)$. Then the central mean subspace exists, is unique, and equals $\mathcal{M}\left(\boldsymbol{\Theta}_{k}\right)$.

By Theorem 1, $\mathcal{M}\left(\boldsymbol{\Theta}_{k}\right) \subset \mathcal{M}(\mathbf{B})$ for any matrix B that satisfies (4). The proof of Corollary 2.1 follows by the fact that $\boldsymbol{\vartheta}_{k+1}=\mathbf{0}$, which implies that $\mathbf{B}$ is of rank $k$, and hence we also have $\mathcal{M}(\mathbf{B}) \subset \mathcal{M}\left(\boldsymbol{\Theta}_{k}\right)$. 


\section{Proof of Theorem 1}

Because $g\left(\mathbf{B}^{T} \mathbf{X}\right)=\tilde{g}\left(\mathbf{B}^{T}(\mathbf{X}-E(\mathbf{X}))\right.$, with $\tilde{g}$ a location shift of $g$, we may assume that $\mathbf{X}$ is centered. Also, since $\left.g\left(\mathbf{B}^{T} \mathbf{X}\right)=g\left(\left(\boldsymbol{\Sigma}^{1 / 2} \mathbf{B}\right)^{T} \boldsymbol{\Sigma}^{-1 / 2} \mathbf{X}\right)\right)$, and because $\mathbf{b} \in \mathcal{M}\left(\boldsymbol{\Sigma}^{1 / 2} \mathbf{B}\right)$ is equivalent to $\boldsymbol{\Sigma}^{-1 / 2} \mathbf{b} \in \mathcal{M}(\mathbf{B})$ we may assume that $\mathbf{X}$ has identity covariance matrix. Set $\mathcal{M}_{0}=\mathcal{M}(\mathbf{B})$.

Consider first the case where $\mathbf{X} \sim N_{d}(\mathbf{0}, \mathbf{I})$. We begin by showing that $\boldsymbol{\vartheta}_{1} \in \mathcal{M}_{0}$. Let $\mathbf{b}_{1} \in \mathcal{M}_{0}, \mathbf{b}_{2} \in \mathcal{M}_{0}^{\perp}$ and set $\mathbf{b}=\mathbf{b}_{1}+\mathbf{b}_{2}$. We will show that

$$
E\left[\left(Y-E\left(Y \mid \mathbf{b}_{1}^{T} \mathbf{X}\right)\right)^{2}\right] \leq E\left[\left(Y-E\left(Y \mid \mathbf{b}^{T} \mathbf{X}\right)^{2}\right]\right.
$$

which implies that the search for minimum in (6) can be confined to $\mathcal{M}_{0}$, and $\boldsymbol{\vartheta}_{1} \in \mathcal{M}_{0}$ follows by the uniqueness of $\boldsymbol{\vartheta}_{1}$. By the fact that the error term $\epsilon=Y-g\left(\mathbf{B}^{T} \mathbf{X}\right)$ satisfies $E(\epsilon \mid \mathbf{X})=0$, it suffices to show that

$$
\operatorname{Var}\left[g\left(\mathbf{B}^{T} \mathbf{X}\right)-\mathrm{E}\left(g\left(\mathbf{B}^{T} \mathbf{X}\right) \mid \mathbf{b}_{1}^{T} \mathbf{X}\right)\right] \leq \operatorname{Var}\left[g\left(\mathbf{B}^{T} \mathbf{X}\right)-\mathrm{E}\left(g\left(\mathbf{B}^{T} \mathbf{X}\right) \mid \mathbf{b}^{T} \mathbf{X}\right)\right] .
$$

Because for any sub- $\sigma$-field $\mathcal{A}$ of $\mathcal{F}(\mathbf{X})$ we have

$$
\operatorname{Var}\left[g\left(\mathbf{B}^{T} \mathbf{X}\right)-\mathrm{E}\left(g\left(\mathbf{B}^{T} \mathbf{X}\right) \mid \mathcal{A}\right)\right]=\mathrm{E}\left[\operatorname{Var}\left(g\left(\mathbf{B}^{T} \mathbf{X}\right) \mid \mathcal{A}\right)\right],
$$

(12) becomes

$$
\mathrm{E}\left[\operatorname{Var}\left(g\left(\mathbf{B}^{T} \mathbf{X}\right) \mid \mathbf{b}_{1}^{T} \mathbf{X}\right)\right] \leq \mathrm{E}\left[\operatorname{Var}\left(g\left(\mathbf{B}^{T} \mathbf{X}\right) \mid \mathbf{b}^{T} \mathbf{X}\right)\right]
$$

By the independence of $\mathbf{B}^{T} \mathbf{X}$ and $\mathbf{b}_{2}^{T} \mathbf{X}$ we have

$$
\operatorname{Var}\left(g\left(\mathbf{B}^{T} \mathbf{X}\right) \mid \mathbf{b}_{1}^{T} \mathbf{X}\right)=\operatorname{Var}\left(g\left(\mathbf{B}^{T} \mathbf{X}\right) \mid \mathbf{b}_{1}^{T} \mathbf{X}, \mathbf{b}_{2}^{T} \mathbf{X}\right) .
$$

Also, by the fact that $\mathcal{F}\left(\mathbf{b}^{T} \mathbf{X}\right)=\mathcal{F}\left(\mathbf{b}_{1}^{T} \mathbf{X}+\mathbf{b}_{2}^{T} \mathbf{X}\right) \subset \mathcal{F}\left(\mathbf{b}_{1}^{T} \mathbf{X}, \mathbf{b}_{2}^{T} \mathbf{X}\right)$ we have

$$
\operatorname{Var}\left(g\left(\mathbf{B}^{T} \mathbf{X}\right) \mid \mathbf{b}_{1}^{T} \mathbf{X}, \mathbf{b}_{2}^{T} \mathbf{X}\right) \leq \operatorname{Var}\left(g\left(\mathbf{B}^{T} \mathbf{X}\right) \mid \mathbf{b}^{T} \mathbf{X}\right), \text { almost surely }
$$

Thus (14) follows from (15) and (16).

Next we will assume that $\mathcal{M}\left(\boldsymbol{\Theta}_{r}\right) \subset \mathcal{M}_{0}$, for some $r=1, \ldots, k-1$, and show that $\boldsymbol{\vartheta}_{r+1} \in \mathcal{M}_{0}$. Let $\mathbf{b}_{1}, \mathbf{b}_{2}$ and $\mathbf{b}$ be as before. By the uniqueness of $\boldsymbol{\vartheta}_{r+1}$, it suffices to show that

$\operatorname{Var}\left[g\left(\mathbf{B}^{T} \mathbf{X}\right)-\mathrm{E}\left(g\left(\mathbf{B}^{T} \mathbf{X}\right) \mid \boldsymbol{\Theta}_{r}^{T} \mathbf{X}, \mathbf{b}_{1}^{T} \mathbf{X}\right)\right] \leq \operatorname{Var}\left[g\left(\mathbf{B}^{T} \mathbf{X}\right)-\mathrm{E}\left(g\left(\mathbf{B}^{T} \mathbf{X}\right) \mid \boldsymbol{\Theta}_{r}^{T} \mathbf{X}, \mathbf{b}^{T} \mathbf{X}\right)\right]$

or, by (13), that

$$
\mathrm{E}\left[\operatorname{Var}\left(g\left(\mathbf{B}^{T} \mathbf{X}\right) \mid \boldsymbol{\Theta}_{r}^{T} \mathbf{X}, \mathbf{b}_{1}^{T} \mathbf{X}\right)\right] \leq \mathrm{E}\left[\operatorname{Var}\left(g\left(\mathbf{B}^{T} \mathbf{X}\right) \mid \boldsymbol{\Theta}_{r}^{T} \mathbf{X}, \mathbf{b}^{T} \mathbf{X}\right)\right] .
$$


Arguing as in (15) and (16),

$$
\begin{aligned}
\operatorname{Var}\left(g\left(\mathbf{B}^{T} \mathbf{X}\right) \mid \boldsymbol{\Theta}_{r}^{T} \mathbf{X}, \mathbf{b}_{1}^{T} \mathbf{X}\right) & =\operatorname{Var}\left(g\left(\mathbf{B}^{T} \mathbf{X}\right) \mid \boldsymbol{\Theta}_{r}^{T} \mathbf{X}, \mathbf{b}_{1}^{T} \mathbf{X}, \mathbf{b}_{2}^{T} \mathbf{X}\right) \\
& \leq \operatorname{Var}\left(g\left(\mathbf{B}^{T} \mathbf{X}\right) \mid \boldsymbol{\Theta}_{r}^{T} \mathbf{X}, \mathbf{b}^{T} \mathbf{X}\right) \text {, almost surely, }
\end{aligned}
$$

which shows (17), and completes the proof of the theorem in the normal case.

Consider now the case where $\mathbf{X} \sim \mathcal{E}_{d}\left(\boldsymbol{\mu}, \boldsymbol{\Sigma}, F_{R}\right)$, i.e., $\mathbf{X}$ has an elliptically symmetric distribution with the representation

$$
\mathbf{X} \stackrel{\mathcal{L}}{=} \boldsymbol{\mu}+R \mathbf{A U}
$$

where the generating random variable $R$ is $>0$ with cumulative distribution function $F_{R}, \mathbf{A}$ is a full rank symmetric $d \times d$ matrix, and $\mathbf{U}$ is independent from $R$ and is uniformly distributed on $S^{d-1}$, the unit sphere in $\mathbb{R}^{d}$. Assume, as before, that the covariates are centered and scaled $\left(\sqrt{d / E\left(R^{2}\right)} \mathbf{A}^{-1}(\mathbf{X}-\right.$ $\boldsymbol{\mu})$ ), so that, using also a well known representation of $\mathbf{U}$ in terms of iid $\mathrm{N}(0,1)$ random variables $Z_{1}, \ldots, Z_{d}$,

$$
\mathbf{X}=\tilde{R}\left(\frac{Z_{1}}{\|\mathbf{Z}\|}, \ldots, \frac{Z_{d}}{\|\mathbf{Z}\|}\right)^{T},
$$

where $\tilde{R}=\sqrt{d / E\left(R^{2}\right)} R$, and $\|\mathbf{Z}\|=\sqrt{Z_{1}^{2}+\cdots+Z_{d}^{2}}$. Write $\mathbf{Z}=\mathbf{Z}_{1}+\mathbf{Z}_{2}$, where $\mathbf{Z}_{1} \in \mathcal{M}_{0}$ and $\mathbf{Z}_{2} \in \mathcal{M}_{0}^{\perp}$, and let $\mathbf{b}_{1}, \mathbf{b}_{2}$ and $\mathbf{b}$ be as before.

First we will show that $\boldsymbol{\vartheta}_{1} \in \mathcal{M}_{0}$ by showing that the result holds conditionally on $\left\|Z_{1}\right\|,\left\|Z_{2}\right\|$, and $R$, almost surely. Because $\mathbf{b}_{2}^{T} \mathbf{Z}$ and $\left\|Z_{2}\right\|$ are independent from $\mathbf{B}^{T} \mathbf{Z}, \mathbf{b}_{1}^{T} \mathbf{Z}$ and $\left\|Z_{1}\right\|$, and $R$ is independent from $\mathbf{Z}$,

$\operatorname{Var}\left(g\left(\mathbf{B}^{T} \mathbf{Z}\right) \mid \mathbf{b}_{1}^{T} \mathbf{Z},\left\|Z_{1}\right\|,\left\|Z_{2}\right\|, R\right)=\operatorname{Var}\left(g\left(\mathbf{B}^{T} \mathbf{Z}\right) \mid \mathbf{b}_{1}^{T} \mathbf{Z}, \mathbf{b}_{2}^{T} \mathbf{Z},\left\|Z_{1}\right\|,\left\|Z_{2}\right\|, R\right)$, a.s.,(19)

since both are equal to $\operatorname{Var}\left(g\left(\mathbf{B}^{T} \mathbf{Z}\right) \mid \mathbf{b}_{1}^{T} \mathbf{Z},\left\|Z_{1}\right\|\right)$, almost surely. Note that (19) involves $g\left(\mathbf{B}^{T} \mathbf{Z}\right)$, not $g\left(\mathbf{B}^{T} \mathbf{X}\right)$. Arguing as in (16), (19) implies

$\operatorname{Var}\left(g\left(\mathbf{B}^{T} \mathbf{Z}\right) \mid \mathbf{b}_{1}^{T} \mathbf{Z},\left\|Z_{1}\right\|,\left\|Z_{2}\right\|, R\right) \leq \operatorname{Var}\left(g\left(\mathbf{B}^{T} \mathbf{Z}\right) \mid \mathbf{b}^{T} \mathbf{Z},\left\|Z_{1}\right\|,\left\|Z_{2}\right\|, R\right)$, a.s.(20)

Since (20) holds for any $g$, and $\|\mathbf{Z}\|=\sqrt{\left\|\mathbf{Z}_{1}\right\|^{2}+\left\|\mathbf{Z}_{2}\right\|^{2}}$ is fixed given $\left\|Z_{1}\right\|$ and $\left\|Z_{2}\right\|$, we also have

$\operatorname{Var}\left(g\left(\tilde{R}\|\mathbf{Z}\|^{-1} \mathbf{B}^{T} \mathbf{Z}\right) \mid \mathbf{b}_{1}^{T} \mathbf{Z},\left\|Z_{1}\right\|,\left\|Z_{2}\right\|, R\right) \leq \operatorname{Var}\left(g\left(\tilde{R}\|\mathbf{Z}\|^{-1} \mathbf{B}^{T} \mathbf{Z}\right) \mid \mathbf{b}^{T} \mathbf{Z},\left\|Z_{1}\right\|,\left\|Z_{2}\right\|, R\right)$, a.s., and hence, by the representation (18) and the equivalence of the $\sigma$-fields

$\mathcal{F}\left(\mathbf{b}^{T} \mathbf{Z},\left\|Z_{1}\right\|,\left\|Z_{2}\right\|, R\right)$ and $\mathcal{F}\left(\mathbf{b}^{T} \mathbf{X},\left\|Z_{1}\right\|,\left\|Z_{2}\right\|, R\right)$, 
for any $\mathbf{b}$, we have

$\operatorname{Var}\left(g\left(\mathbf{B}^{T} \mathbf{X}\right) \mid \mathbf{b}_{1}^{T} \mathbf{X},\left\|Z_{1}\right\|,\left\|Z_{2}\right\|, R\right) \leq \operatorname{Var}\left(g\left(\mathbf{B}^{T} \mathbf{X}\right) \mid \mathbf{b}^{T} \mathbf{X},\left\|Z_{1}\right\|,\left\|Z_{2}\right\|, R\right)$, a.s. $(21)$

Relation (21) implies that (14), and hence that $\boldsymbol{\vartheta}_{1} \in \mathcal{M}_{0}$, holds conditionally on $\left\|Z_{1}\right\|,\left\|Z_{2}\right\|$ and $R$, a.s. Thus, it also holds unconditionally. The proof of the induction step uses arguments similar to the above and those used in the induction step in the normal case.

\section{Methodological Overview and Open Problems}

In this section contains a partial review of available methods for fitting the SI and MI models and highlights some open problems.

In addition to the least squares method Brillinger (1983), three other types of methods have been suggested for estimating $\boldsymbol{\beta}$ in the SI model (1). The first type is based on Stoker's (1986) observation that the expectation of the gradient $\nabla(\mu(\mathbf{X}))$ is a scalar multiple of $\boldsymbol{\beta}$; such average derivative estimation (ADE) methods include Powel et al. (1989), Härdle and Stoker (1989), the direct estimation approach of Hristache et al. (2001), and the outer product of gradients (OPG) method of Xia et al. (2002). The second type includes Ichimura (1993), who termed the approach semiparametric least squares (SLS) estimation, Hall (1989), Härdle et al. (1993), Liang et al. (2010) who also use penalized semiparametric least squares for simultaneous estimation and variable selection and Cui et al. (2011); related is the minimum average variance estimation (MAVE) method of Xia et al. (2002). The third type includes methods that evolved from the sliced inverse regression methodology of $\mathrm{Li}$ (1991) which requires the same "linearity condition" as the least squares method. Though some of the above references pertain only to the SI model, the same three types of methods have also been applied for estimating the parameters of the MI model (1); see also Cook and Li (2002) and Li et al. (2004).

Of the methods mentioned for estimating $\boldsymbol{\beta}$ in (1) only SLS-type methods estimate the first projective direction (which is always a well defined quantity) regardless of whether or not the SI model holds. For example, though average derivatives are statistically meaningful (Samarov, 1993), and have been studied on their own right (Chaudhuri et al., 1997), projection on the vector of average derivatives has no clear interpretation if (1) does not hold. Moreover, of the papers that use the SLS estimation method, only 
Hall (1989) considers the asymptotic properties of the SLS estimator without assuming the SI model. In particular, asymptotic distribution theory for the estimated projective directions under the PPR model (2) does not exist. In recent work, Akritas (2016) derives the asymptotic distribution of the first projective direction without assuming the SI model. The asymptotic theory for the joint projective directions under PPMI models, including the subclass that includes the PPR model, remains an open problem.

Akritas, M. G. (2016). Asymptotic Theory for the First Projective Direction. Annals of Statistics, in press.

Brillinger, D. R. (1983). A generalized linear model with "Gaussian" regressor variables. In Bickel, P.J., Doksum, K.A., and Hodges, J.L., eds. A festschrift for Erich L. Lehmann, pages 97-114. Wordsworth International Group, Belmont, CA.

Chaudhuri, P., Doksum, K. and Samarov, A. (1997). On average derivative quantile regression. Annals of Statistics, 25, 715-744.

Cook, R.D. and Li, B. (2002). Dimension Reduction for Conditional Mean in Regression. Annals of Statistics, 30, 455-823.

Cui, X., Härdle, W. K. and Zhu, L. (2011). The EFM approach for singleindex models. Annals of Statistics, 39, 1658-1688.

Friedman, J.H. and Stuetzle, W. (1981). Projection pursuit regression. Journal of the American Statistical Association, 76, 817-823.

Hall, P. (1989). On projection pursuit regression. Annals of Statistics, 17, $573-588$.

Härdle, W., Hall, P. and Ichimura, H. (1993). Optimal smoothing in singleindex models. Annals of Statistics, 21, 157-178.

Härdle, W. and Stoker, T.M. (1989). Investigating Smooth Multiple Regression by the Method of Average Derivatives. Journal of the American Statistical Association, 84, 986-995

Hristache, M., Juditski, A. and Spokoiny, V. (2001). Direct estimation of the index coefficients in a single-index model. Annals of Statistics, 29, 595-623. 
Huber, P. J. (1985). Projection pursuit (with discussion). Annals of Statistics, 13, 435-525.

Ichimura, H. (1993). Semiparametric least squares (SLS) and weighted SLS estimation of single-index models. Journal of Econometrics 58, 71-120.

Li B., Cook R.D. and Chiaromonte F. (2004). Dimension reduction for the conditional mean in regressions with categorical predictors. Annals of Statistics, 30, 1636-1668.

Li, K. C. (1991). Sliced Inverse Regression for Dimension Reduction (with discussion). Journal of the American Statistical Association, 86, 316-342.

Liang, H, Liu, X., Li, R., and Tsai, C.-L. (2010). Estimation and testing for partially linear single-index models. Annals of Statistics, 38, 3811-3836.

Powell, J.L., Stock, J.H. and Stoker, T.M. (1989). Semiparametric estimation of index coefficients. Econometrica, 57, 1403-1430.

Samarov, A.M. (1993). Exploring regression structure using nonparametric functional estimation. Journal of the American Statistical Association, 88, 836-847.

Stoker, T. M., (1986). Consistent estimation of scaled coefficients. Econometrica, 54, 1461-1481.

Stone, C. J. (1985). Additive regression and other nonparametric models. Annals of Statistics, 13, 689-705.

Xia, Y. (2008). A Multiple-Index Model and Dimension Reduction. Journal of the American Statistical Association, 103, 1631-1640.

Xia, Y., Tong, H., Li, W. K. and Zhu, L. (2002). An adaptive estimation of dimension reduction space (with discussions). J. R. Statist. Soc. Ser. B, $64363-410$. 